Universe: An Introduction to Astronomy, by Harry L. Shipman (Houghton Mifflin: Boston and London) and Cosmic Evolution: An Introduction to Astronomy, by G. B. Field, G. L. Verschuur and C. Ponnamperuma (Houghton Mifflin: Boston and London). They are all the same size; one is red, the other blue, the third brown; they contain the regulation collection of colour plates (where would one be without NASA and the Hale Observatory?). They are beautifully illustrated, well indexed, glossaried and spattered with review questions, thought questions, chapter summaries, and the like. They each have their "special way of approaching the subject'. Chapman uses the historical angle starting from Earth and the view Earth dwellers have of the sky and moving out to the Moon, Sun, planets, stars, Galaxy and Universe. Shipman states that he differs from similar texts by stressing contemporary astronomy - "the events that readers have probably heard about from the media" and by indulging in a detailed intercomparison of the 'geological' processes that have taken place on different planets. Field, Verschuur and Ponnamperuma concentrate on the evolution of the Universe from the primordial big bang, through the origin of galaxies, stars and planets to the emergence of intelligent life on Earth and the search for this life elsewhere. Even with these "different approaches' in mind I was continually struck by the overall similarities. The differences are at best marginal and in most cases simply seem to be a difference in the ordering, much more than in content. In my experience it is a very rare lecture course that starts at the beginning of a textbook and methodically works its way through chapter by chapter, so the ordering is fairly immaterial. So I have a problem, which one should I recommend? I shudder to suggest that you should resort to 'eeny, meeny, miney, mo' but this is one way out. The other is simply to buy the cheapest.

My final book is Exploration of the Solar System by William J. Kaufmann III (Macmillan: New York; Collier Macmillan: London; $£ 0.00$ ). A book mainly about planets with a little on stars and a sister to Kaufmann's 1977 book Astronomy: The Structure of the Universe, which was mainly about stars with a little on planets. Kaufmann's books are aimed at the same non-mathematical audience as the three mentioned above. In Exploration of the Solar System one feels that Kaufmann has room to spread himself. $\mathrm{He}$ is not restricted by space, he studies the problems, the spacecraft, the explorations, the data, the planetary features and models in detail and with thoroughness.

In general I was extremely impressed by the high standards of these books. Writing an astronomical textbook is no easy task because topic after topic is in a state of rapid development. To quote
R. D. Chapman "it is inevitable that some parts of any book will be out of date by the time the ink has dried". It must be frustrating to write a line such as "ratio of the mass of Pluto to that of the Earth $=0.1(?) "$ months before the mass was accurately measured due to the discovery

\section{Mathematical cosmology}

Mathematical Cosmology. By P. T. Landsberg and D. A. Evans. Pp. 306. (Clarendon/Oxford University Press: Oxford, 1978.) $£ 6.95$.

Although the number of students taking formal courses in cosmology is tiny, most physical scientists find room in their activities to read up on it from time to time. The stumbling block to penetrating the technical literature is usually the general theory of relativity, with its mind bending concepts of curved spacetime, event horizons and singularities, not to mention punishing (as well as non-linear) mathematics. The solution to this barrier is, according to P. T. Landsberg and D. A. Evans in their Mathematical Cosmology, a "stage II" book, defined in their introduction to be one which goes beyond the broad qualitative outlines, but uses only simple mathematical arguments. And this means no general relativity. Although other books, such as Michael Berry's Principles of Cosmology and Gravitation (Cambridge University Press: Cambridge, 1976), also attempt this, Professors Landsberg and Evans give probably the first comprehensive and systematic non-relativistic treatment of cosmology.

Students familiar with Bondi's little book Cosmology will know how fairly elementary Newtonian ideas can lead effortlessly to the main features of relativistic cosmological models. The authors of Mathematical Cosmology follow the same path, but in a much more elaborate and detailed

\section{Vibrations and waves}

Vibrations and Waves in Physics. By I. G. Main. Pp. 336. (Cambridge University Press: Cambridge and London, 1978.) Hardback $£ 17.50$; paperback $£ 4.95$.

THIS is an excellent book which can be thoroughly recommended either for use as a text for an undergraduate course introducing the student to many of the widely-pervading notions connected with vibrations and waves, or indeed for anyone who feels the need and observation of a satellite. But still the job needs to be done and it is refreshing to see how well it is done time after time. $\square$

David W. Hughes is Lecturer in Astronomy and Physics at the University of Sheffield, UK.

fashion. All the basic conceptual structure is kept simple, and individual chapters can be read in isolation without difficulty. The standard Friedmann models are thoroughly discussed, and even the steady state theory gets a mention. Each chapter is terminated with a set of questions, the answers to which are provided.

Eventually, perhaps inevitably, general relativity refuses to be excluded. It starts to intrude during the discussion of light propagation, and the appearance of distant astronomical objects. However, in this limited context, without the field equations of the dynamics of the spacetime itself, there is nothing terribly formidable about it, and students should be able to follow the lucid discussion easily. When horizons are finally discussed, the approach is physical rather than geometrical, and indeed, this is true of the book as a whole.

For scientists and students with a serious interest in cosmology who do not want to invest a lot of time learning differential geometry and topology, Mathematical Cosmology gives an excellent, clearly written and coherent account of the standard theory which is very economical on intellectual investment. It is also economical on the pocket, because at $£ 6.95$, it is rather cheap for a 306-page textbook. For students taking cosmology the hard way, it is still worthwhile background reading, and of course most of the results are identical to those of the relativistic treatment.

Paul Davies

Paul Davies is Senior Lecturer in the Department of Mathematics at King's College, University of London, $U K$.

to broaden his knowledge in this sphere. The material covered starts with free vibrations and continues through forced vibrations, anharmonic and coupled vibrations, to nondispersive waves. After a short chapter on Fourier theory the author looks at dispersion, water waves, electromagnetic waves, and de Broglie waves, and finally covers basic ideas in the reflection, refraction, and diffraction of waves.

The book is well and carefully constructed. An example of this is the useful flow diagram near the front, showing clearly which are the parts containing the coherent development 